\title{
How Deposition Parameters Affect Phase Formation in Metals
}

\author{
Tahir Mehmood ${ }^{1, ~ *, ~ A i m a n ~ M u k h t a r ~}{ }^{1}$, Babar Shahzad Khan², Wu Kaiming ${ }^{1}$ \\ ${ }^{1}$ The State Key Laboratory of Refractories and Metallurgy, Hubei Province Key Laboratory of Systems Science in Metallurgical Process, \\ International Research Institute for Steel Technology, Wuhan University of Science and Technology, Wuhan, P. R. China \\ ${ }^{2}$ Department of Physics, Govt. College Women University, Sialkot, Punjab, Pakistan
}

Email address:

tahir10621@yahoo.com (T. Mehmood),wukaiming@wust.edu.cn (Wu Kaiming)

${ }^{*}$ Corresponding author

To cite this article:

Tahir Mehmood, Aiman Mukhtar, Babar Shahzad Khan, Wu Kaiming. How Deposition Parameters Affect Phase Formation in Metals. American Journal of Applied Chemistry. Vol. 4, No. 5, 2016, pp. 192-200. doi: 10.11648/j.ajac.20160405.16

Received: August 6, 2016; Accepted: September 19, 2016; Published: October 13, 2016

\begin{abstract}
To understand the mechanism for formation of fcc-cobalt nanowires in electrodeposition, we have systematically studied the effect of deposition potential, $\mathrm{pH}$, and deposition temperature on the formation of fcc Co nanowires by X-ray diffraction (XRD), transmission electron microscope (TEM) and scanning electron microscope (SEM). The Co nanowires deposited at the potential of $-1.6 \mathrm{~V}$ are pure hcp phase. When increasing the value of potential to $-2.0 \mathrm{~V}$, there are hep Co and fcc Co crystals in the deposited nanowires. The fraction of fcc Co crystals in the nanowires increases with increasing the potential value. At $-3.0 \mathrm{~V}$, the nanowires are pure fcc $\mathrm{Co}$. The $\mathrm{pH}$ of the solution has little effect on formation of fcc Co nanowires. We have also seen that high concentration and low temperature favors fcc phase whereas low concentration and high temperature favors hcp phase. However, at $35^{\circ} \mathrm{C}$ the co-occurrence of hcp and fcc phases were also observed. These experimental results can be explained by the classical electrochemical nucleation theory. The formation of fcc Co crystals can be attributed to smaller critical clusters formed at a higher potential value since the smaller critical clusters favor formation of fcc nuclei.
\end{abstract}

Keywords: Nanostructure, Electrodeposition, Fcc Cobalt, Crystal Growth

\section{Introduction}

The metallic cobalt (Co) is an hcp (hexagonal close packing) phase at temperatures below $420^{\circ} \mathrm{C}$ and an fcc (face-centered cubic) phase at temperatures above $420^{\circ} \mathrm{C}$. However, the high temperature phase, fcc $\mathrm{Co}$, can be formed in electrodeposition at ambient temperature. [1-3] A lot of studies have been done on experimental conditions of forming fcc $\mathrm{Co}$ in electrodeposition at ambient temperature. These studies show that the formation of fcc $\mathrm{Co}$ is favored at lower $\mathrm{pH}$ of electrolyte [1, 4-7] and at higher potentials. [2, 8, 9] Studies on the mechanism of forming fcc Co in electrodeposition. During the process of electrodeposition, by adjusting deposition conditions such as the $\mathrm{pH}$ value [10], pulse electrodeposition [11, 12], deposition potential [13], deposition current density [14], and deposition temperatures [15], the microstructure of Co nanowire was adjusted accordingly. However, have not received much attention and only a few reports are found on the dependence of crystal orientation of cobalt nanowires with bath temperature [16], and electrolyte concentration [17]. For example, Nakahara and Mahajan argued: [1] metastable fcc Co hydride could form during electrodeposition at low $\mathrm{pH}$; after the deposition, the fcc Co hydride phase can be decomposed and partially transformed to hcp Co because of the rapid out-diffusion of hydrogen. This can explain their experimental observation that the Co film prepared at low $\mathrm{pH}$ contains not only fec Co but also hep Co. [1] Studies of Wang et al [8] and Huang et al [9] show that the single crystalline Co nanowires are hep phase at the lower deposition potential of about $-1.5 \mathrm{~V}$, and fcc phase at the higher deposition potential of $-3.0 \mathrm{~V}$. Cohen-Hyams et al also observed fcc Co in the film deposited at high potentials. [2] Wang et al, [8] Huang et al [9] and Cohen-Hyams et al [2] explained the formation of fcc Co using the view of Nakahara and Mahajan. [1] We disagree with the view of Nakahara and Mahajan, based on three reasons. First, XRD results obtained by Wang et al and Huang et al show that the nanowires deposited at $-3.0 \mathrm{~V}$ are pure fcc 
Co, [8, 9] indicating that no decomposition of fcc Co hydride phase into hcp Co phase occurs. This cannot be explained by the model proposed by Nakahara and Mahajan. [1] Second, a lot of TEM (transmission electron microscope) and XRD (X-ray diffraction) studies on the Co fine particles prepared by the vacuum deposition (vapor and sputtering depositions) show that the structure of Co fine particles depends on their sizes. [18-22] The Co particles are fcc structure for smaller sizes and hep structure for larger sizes. $[19,22]$ Third, there is only a very small amount of hydrogen molecules in the vacuum chamber for deposition of Co particles. This indicates that hydrogen plays no role in forming fec Co particles in vacuum deposition.

In this paper, an alternative explanation of why fcc Co nanowires form in electrodeposition at ambient temperature can be seen. Firstly, some systematic results about the effect of deposition potential, $\mathrm{pH}$, deposition temperature and electrolytic cell concentration on the crystal structure of $\mathrm{Co}$ nanowires are discussed and then account for why $\mathrm{Co}$ nanowires are hcp structure at a lower potential value and fcc structure at a higher potential value and also show that how phase changes from fcc to hcp Co with respect to concentration and temperature.

\section{Experimental Details}

The detailed experimental procedure for preparation of the porous anodic alumina oxide (AAO) templates has been given in our previous paper. [23] Here we briefly describe the experimental procedure. Firstly, high-purity aluminum foils (99.999\%) were degreased in acetone and then were annealed in a vacuum of $10^{-5}$ Torr at $500^{\circ} \mathrm{C}$ for $5 \mathrm{~h}$ to remove the mechanical stress. Secondly, the aluminum foils were anodized in a $0.3 \mathrm{M} \mathrm{H}_{2} \mathrm{C}_{2} \mathrm{O}_{4}$ solution at $2^{\circ} \mathrm{C}$ for $6 \mathrm{~h}$. After removing the alumina layer formed in the anodization in a mixture of phosphoric acid (6 wt\%) and chromic acid (1.8 $\mathrm{wt} \%)$, the aluminum foils were anodized again at the same conditions as the first anodization for $12 \mathrm{~h}$. The templates that experienced the above two-step anodization were etched in a saturated $\mathrm{CuCl}_{2}$ solution to remove the remaining aluminum on the back side. Thirdly, the alumina barrier layer was then dissolved in a $5 \mathrm{wt} \%$ phosphoric acid solution at $40^{\circ} \mathrm{C}$. The templates obtained by the above method havethe cylindrical and hexagonally arranged pores of about $50 \mathrm{~nm}$ in diameter. Finally, a gold $(\mathrm{Au})$ film was sputtered onto the back side of the templates to serve as the working electrode.

The electrolyte used in this study was a mixture of $\mathrm{CoSO}_{4} \cdot 7 \mathrm{H}_{2} \mathrm{O}$ having three different concentrations $(0.71 \mathrm{M}$, $0.53 \mathrm{M}$, and $0.35 \mathrm{M})$ and $\mathrm{H}_{3} \mathrm{BO}_{3}(0.68 \mathrm{M})$ in aqueous solutions. Direct current electrodeposition was conducted in a two-electrode cell under different deposition potentials, $\mathrm{pH}$, temperature and concentration respectively. The area of the working electrode for growth of nanowires was $0.608 \mathrm{~cm}^{2}$ $\left(=0.25 \pi(0.88 \mathrm{~cm})^{2}\right)$ and the area of the graphite counter electrode was $14.7 \mathrm{~cm}^{2}(=4.2 \mathrm{~cm} \times 3.5 \mathrm{~cm})$. The adjustment of $\mathrm{pH}$ of the electrolyte was done by adding $1 \mathrm{M} \mathrm{H}_{2} \mathrm{SO}_{4}$ solution.

The Co nanowires were analyzed by $\mathrm{X}$-ray diffraction
(XRD, Y-2000) with $\mathrm{CuK}_{\alpha}$ radiation $(\lambda=0.154178 \mathrm{~nm})$. Before XRD measurements the sputtered $\mathrm{Au}$ film was mechanically polished away. The deposited Co nanowires were also analyzed by scanning electron microscope (SEM, JEOL JSM-6700F) and transmission electron microscope (TEM, JEOL JEM-2010). In order to perform SEM observations, the AAO template was partly dissolved with a 5 $\mathrm{wt} \% \mathrm{NaOH}$ solution, and then carefully rinsed with deionized water for several times. For TEM observations, the AAO was completely dissolved in a $5 \mathrm{wt} \% \mathrm{NaOH}$ solution, then rinsed with deionized water several times, and finally dispersed in absolute ethanol by ultrasonic.

\section{Results}

\subsection{For Deposition Potential and $\mathrm{pH}$}

Fig. 1 show the XRD patterns of Co nanowires deposited in the solution with a $\mathrm{pH}$ of 2.5 at four different potentials of -1.6 , $-2.0,-2.5$ and $-3.0 \mathrm{~V}$. The XRD data were collected from the top side of nanowires. The nanowires of Co prepared at $-1.6 \mathrm{~V}$ have a strong peak at $2 \theta=41.6$ degrees and this peak can be unambiguously attributed to the hep Co (1010) plane diffraction. The nanowires of Co prepared at $-3.0 \mathrm{~V}$ have a strong peak at $2 \theta=75.6$ degrees.

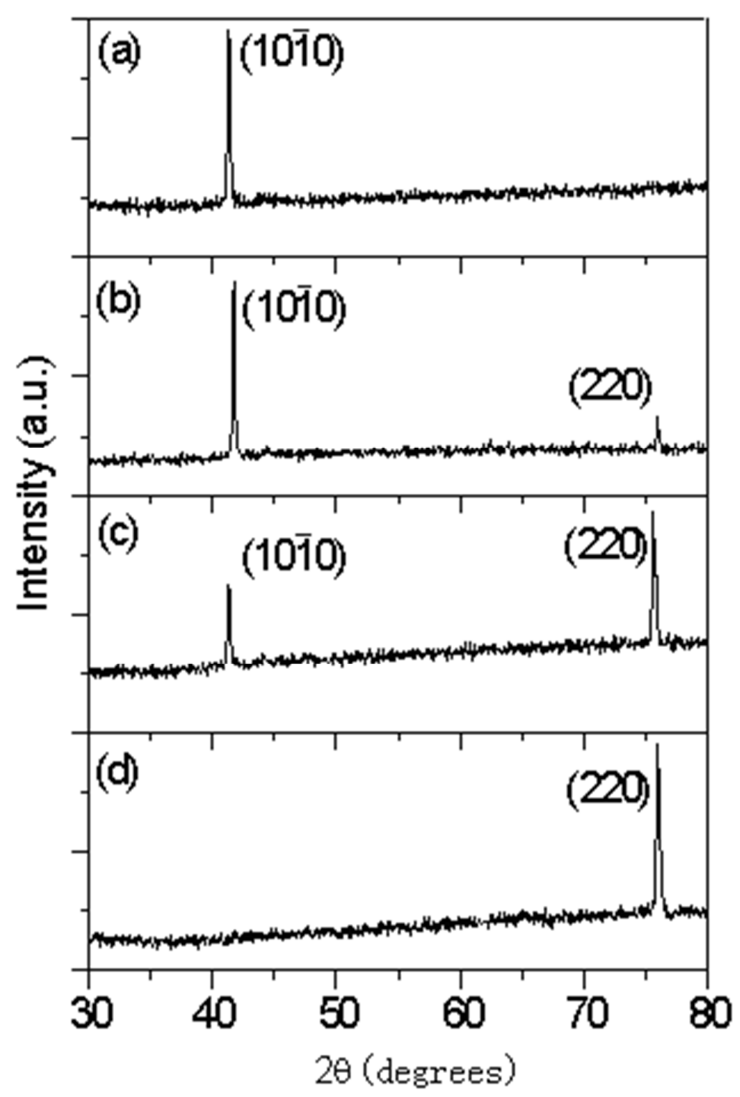

Figure 1. XRD patterns of Co nanowires deposited at different potentials: (a) $-1.6 \mathrm{~V}$, (b) $-2.0 \mathrm{~V}$, (c) $-2.5 \mathrm{~V},(\mathrm{~d})-3.0 \mathrm{~V}$.

The peak at $2 \theta=75.6$ degrees is difficult to index from 
XRD measurements since the hcp Co (1120) and fcc Co (220) planes have the same diffraction peak position at $2 \theta=75.6$ degrees. Since single crystal electron diffraction pattern can be used to distinguish crystal structure, [24] we have performed TEM observations on the Co nanowires deposited at $-3.0 \mathrm{~V}$ in order to identify this peak. Fig. 2 gives the TEM image and electron diffraction pattern of a Co nanowire deposited at -3.0V.
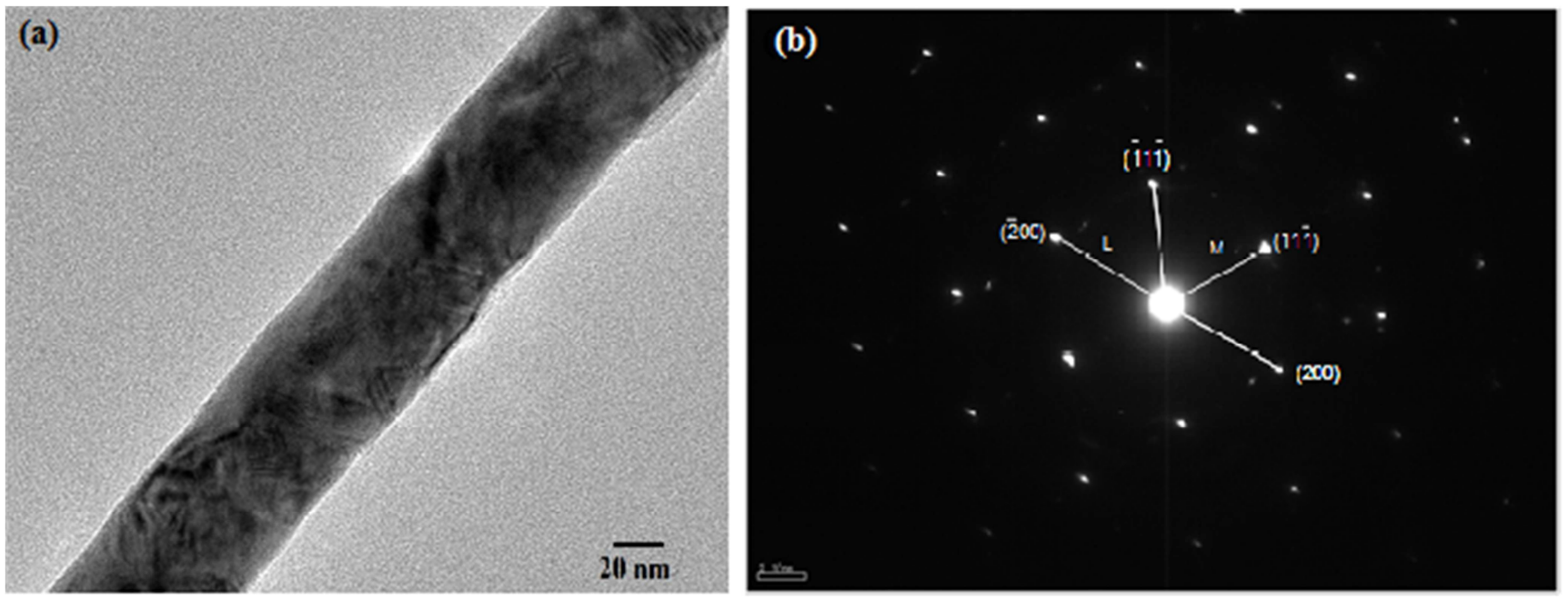

Figure 2. TEM image (a) and electron diffraction pattern (b) of a Co nanowire deposited in the solution of pH 2.5 at $-3.0 \mathrm{~V}$.

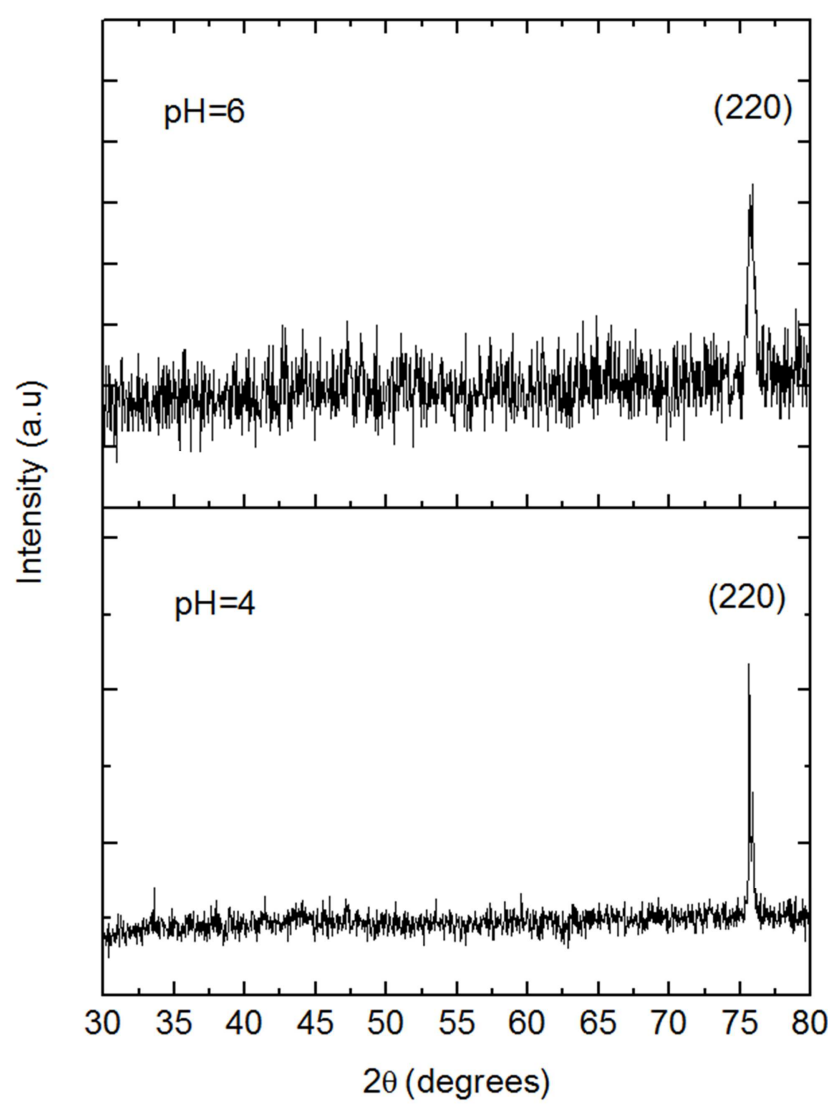

Figure 3. XRD patterns of $f c c$ Co nanowires deposited at $-3.0 \mathrm{~V}$ in the solutions of $\mathrm{pH} 4.0$ and $\mathrm{pH}$ 6.0.

The observed electron diffraction pattern is the same as the standard fec crystal diffraction pattern in the case of the incident electron beam direction being $<110>$. Moreover, the observed ratio of the spot spacings of $L$ to $M(1.163)$ is in agreement with the ratio $(2 / \sqrt{3}=1.155)$ in the standard diffraction pattern. Therefore, the Co nanowires deposited at $-3.0 \mathrm{~V}$ are an fcc phase. Our TEM results are in agreement with those obtained by Wang et al [8] and Huang et al [9]. Using HRTEM, Wang et al and Huang et al have demonstrated that the nanowires of $\mathrm{Co}$ deposited at $-3.0 \mathrm{~V}$ are fcc single crystalline, thus attributing the peak at $2 \theta=75.6$ degrees to the diffraction of fcc (220) plane. After indexing the peak at $2 \theta=75.6$ degrees, the effect of deposition potentials on the formation of fcc Co can be seen. It can be seen from Fig. 1 that the nanowires deposited at $-1.6 \mathrm{~V}$ are pure hep Co. The Co nanowires deposited at $-2.0 \mathrm{~V}$ contain hep and fcc crystals; and the fraction of hep crystals is much more than that of fcc crystals. The fraction of fcc crystals increases with increasing the potential value. The Co nanowires deposited at $-3.0 \mathrm{~V}$ are pure fec Co.

Previous studies show that, in the case of the electrodeposited Co films, low $\mathrm{pH}(<2.4)$ favors formation of fcc Co while high $\mathrm{pH}(>2.9)$ favors hcp Co. In order to understand the effect of $\mathrm{pH}$ on the formation of fcc Co nanowires, Co nanowires deposited at $-3.0 \mathrm{~V}$ in the solution of $\mathrm{pH} 4.0$ and $\mathrm{pH}$ 6.0, respectively. Fig. 3 shows the XRD patterns of $\mathrm{Co}$ nanowires deposited at $-3.0 \mathrm{~V}$ in the solution with $\mathrm{pH}$ of 4.0 and 6.0. No peak was observed due to the hep Co on the patterns, indicating that the $\mathrm{pH}$ of the solution has little effect on the formation of fcc Co nanowires at the $\mathrm{Au}$-coated AAO template for the $\mathrm{pH}$ range studied.

In order to get a complete understanding of the effect of deposition potential and $\mathrm{pH}$ on formation of Co nanowires, experimental results of Co nanowires, which were obtained by other researchers and us, are aggregated into Table 1. One can see three points from Table 1. First, high (low) deposition potential favors formation of fcc (hcp) Co nanowires. Second, the $\mathrm{pH}$ effect does not occur in deposition of Co nanowires. 
Third, the experimental observations of forming fcc Co nanowires at high $\mathrm{pH}$ (4.0-6.5) cannot be explained by the

mechanism of Nakahara and Mahajan.

Table 1. The effect of deposition potential and pH on crystal structure of Co nanowires. S represents the single-crystalline and P the polycrystalline.

\begin{tabular}{llll}
\hline Co nanowires & deposition potential (or current density) & pH & references \\
\hline Pure hep Co (S) & $-1.5 \mathrm{~V},-1.45 \mathrm{~V}$ & $4.0,5.5$ & Huang et al, [9] Wang et al [8] \\
Pure fcc Co (S) & $-3.0 \mathrm{~V}$ & $2.0,5.5$ & Huang et al, [9] Wang et al [8] \\
Pure fcc Co (P) & 2.5 or $40 \mathrm{~mA} / \mathrm{cm}^{2}$ & $3.0,6.0,6.5$ & Cho et al [25] \\
Pure hcp Co (S) & $-1.6 \mathrm{~V}$ & 2.5 & this work \\
Pure fcc Co (S) & $-3.0 \mathrm{~V}$ & $2.5,4.0,6.0$ & this work \\
\hline
\end{tabular}

Fig. 4 displays SEM images of Co nanowires deposited at $-1.6 \mathrm{~V}$ in the solution of $\mathrm{pH} 2.5$ (Fig. $4 \mathrm{a}$ ) and at $-3.0 \mathrm{~V}$ in the solution of pH 4.0 (Fig. 4b) and pH 6.0 (Fig. 4c) separately.

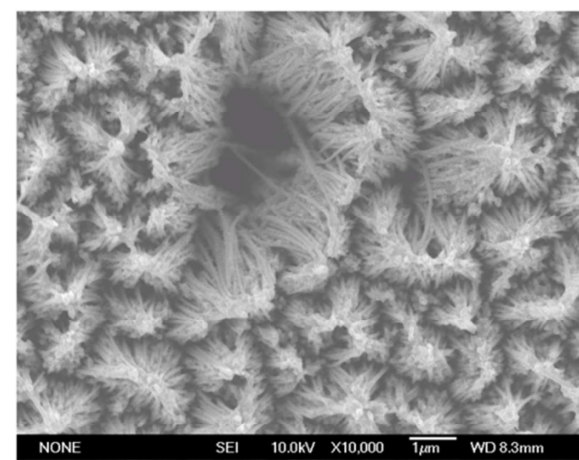

(a)

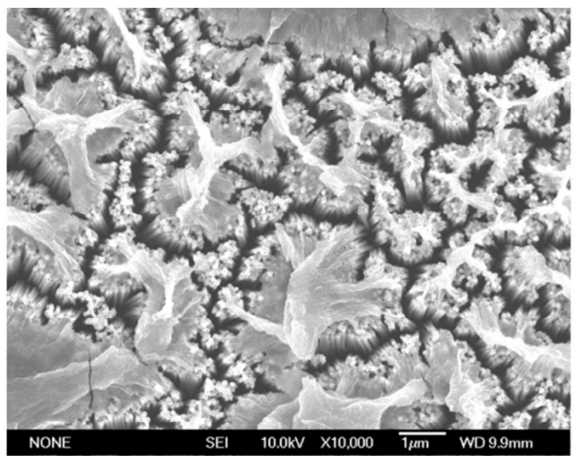

(b)

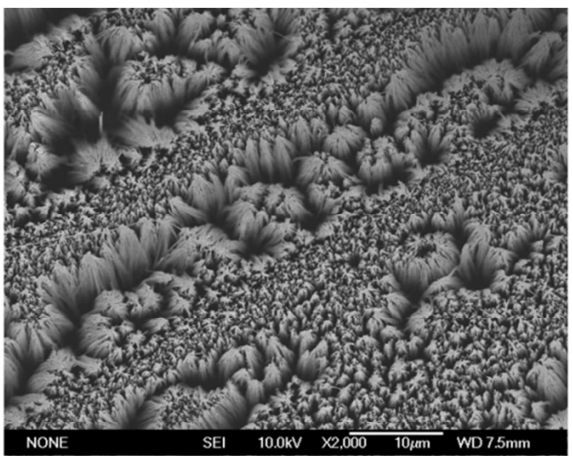

(c)

Figure 4. SEM images of Co nanowires deposited at $-1.6 \mathrm{~V}$ and $\mathrm{pH} 2.5$ (a), at $-3.0 \mathrm{~V}$ and $\mathrm{pH} 4.0(\mathrm{~b})$, at $-3.0 \mathrm{~V}$ and $\mathrm{pH} 6.0$ (c).

The diameter of the Co nanowires $(\sim 50 \mathrm{~nm})$ is the same as that of the pores of AAO template $(\sim 50 \mathrm{~nm})$, indicating that the cylindrical pores of the AAO template were fully filled with Co atoms during electrodeposition.

\subsection{For Deposition Temperature and Electrolytic Cell Concentration}

Fig. 5 shows the XRD patterns of Co nanowires deposited in the solution with concentration of $0.71 \mathrm{M}$ at $\mathrm{pH}$ of 2.5 and at six different temperatures $\left(5^{\circ} \mathrm{C}, 15^{\circ} \mathrm{C}, 25^{\circ} \mathrm{C}, 35^{\circ} \mathrm{C}, 45^{\circ} \mathrm{C}\right.$, and $60^{\circ} \mathrm{C}$ ) under deposition potential of $-3 \mathrm{~V}$.

The XRD patterns were collected from the top side of nanowires. The coexistence of both hep and fcc cobalt phases were seen. It is found that the crystal structure of Co nanowires lies on the deposition conditions. When electrodepostion is performed in a same electrolytic cell but at different temperatures, the phase changed from fcc (at low temperature) to hcp (at high temperature). Fig. 5 (a, b, and c) represent that at low temperatures $5^{\circ} \mathrm{C}, 15^{\circ} \mathrm{C}$, and $25^{\circ} \mathrm{C}$, the well-known fcc structure of $\mathrm{Co}$ has been deposited, respectively, which is stable at temperatures above $422^{\circ} \mathrm{C}[10]$, while increasing the temperature to $35^{\circ} \mathrm{C}-60^{\circ} \mathrm{C}$ both hcp and fcc structures exist there (see Fig. 5 (d and e)). From the figure one can also observe at $25^{\circ} \mathrm{C}$ the phase for deposited $\mathrm{Co}$ nanowires is fcc and result agreed with the result of wang et al and Huang et al $[13,26]$.

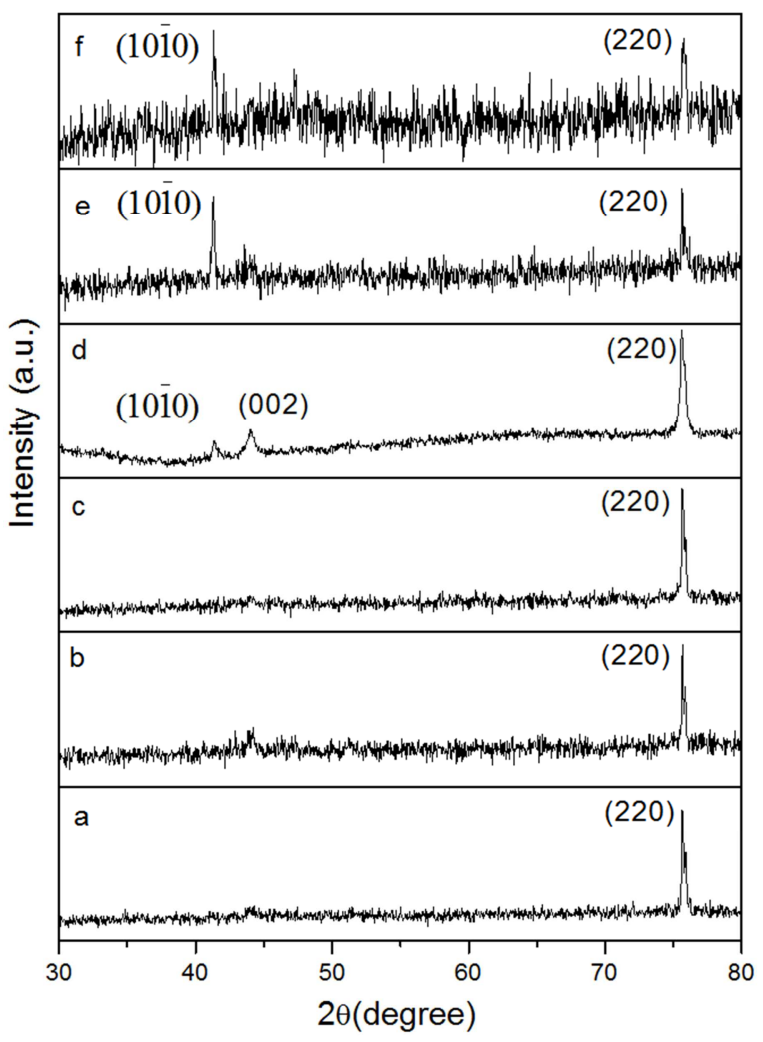

Figure 5. XRD pattern for electrochemically deposited Co nanowires in the pores of $A A O$ template at different temperatures having concentration of $0.71 \mathrm{M}$, a) $\left.5^{\circ} \mathrm{Cb}\right) 15^{\circ} \mathrm{C}$, c) $25^{\circ} \mathrm{C} \mathrm{d}$ ) $45^{\circ} \mathrm{C}$, and e) $60^{\circ} \mathrm{C}$. 
Fig. 6 illustrates the XRD patterns of Co nanowires deposited in electrolytic cell having solution with concentration of $0.53 \mathrm{M}$ (cobalt sulfate) and $\mathrm{pH}$ of 2.5 . The deposition is performed at five different temperatures $\left(15^{\circ} \mathrm{C}\right.$, $25^{\circ} \mathrm{C}, 35^{\circ} \mathrm{C}, 45^{\circ} \mathrm{C}$, and $60^{\circ} \mathrm{C}$ ) at deposition potential of $-3 \mathrm{~V}$. From the Fig. 6 (a, c, d, and e), the fcc structure is seen at low temperature of $15^{\circ} \mathrm{C}$ and $25^{\circ} \mathrm{C}$, while the universal hcp structure for the electrodeposited $\mathrm{Co}$ is obtained at temperatures of $45^{\circ} \mathrm{C}$, and $60^{\circ} \mathrm{C}$, respectively.

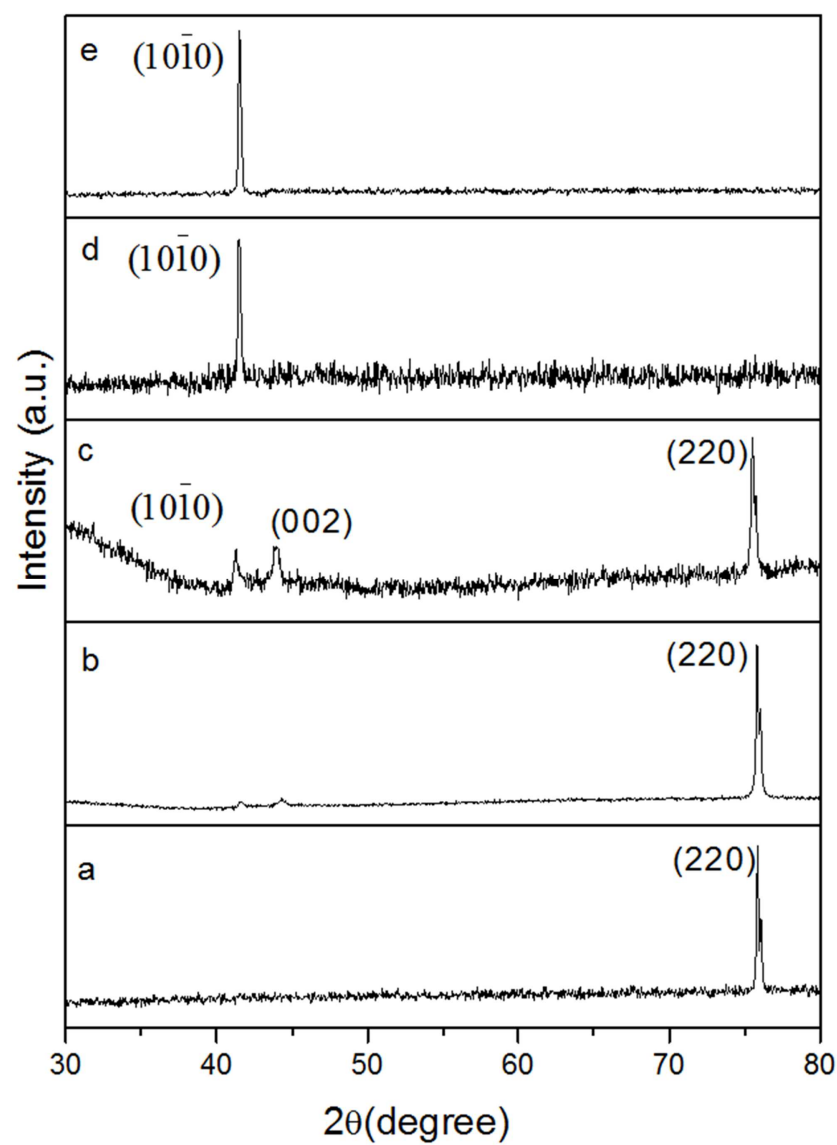

Figure 6. XRD pattern for electrochemically deposited Co nanowires in the pores of $A A O$ template at different temperatures having concentration of $0.53 \mathrm{M}$, a) $\left.15^{\circ} \mathrm{C} \mathrm{b}\right) 25^{\circ} \mathrm{C}$, c) $35^{\circ} \mathrm{C} \mathrm{d}$ ) $45^{\circ} \mathrm{C}$, and e) $60^{\circ} \mathrm{C}$.

At temperature of $35^{\circ} \mathrm{C}$ the coexistence of hcp $(10 \overline{1} 0),(002)$ and fcc (220) diffraction peaks present that phase is being changed (see Fig. 6 (b)).

To find out the concentration effect with change in temperature Co nanowires were also deposited in a cell containing $0.35 \mathrm{M}$ concentration of cobalt sulfate at $\mathrm{pH}$ of 2.5 . $\mathrm{XRD}$ results for this cell at four different temperatures $\left(25^{\circ} \mathrm{C}\right.$, $35^{\circ} \mathrm{C}, 45^{\circ} \mathrm{C}$, and $60^{\circ} \mathrm{C}$ ) are presented in Fig. 3. From the Fig. 7 (a) the diffraction peaks at $2 \theta=41.5$, and 75.6 degrees confirm the existence of hcp and fcc structure at temperature $25^{\circ} \mathrm{C}$. At temperature $35^{\circ} \mathrm{C}$ there is a little increase in the intensity of $(10 \overline{10})$ which shows that some nanowires grow along [10 10$]$ direction and others along [220] direction. It can be seen from the Fig. 7 (c, and d) that further increases in temperature give the pure hcp phase. There is only a single peak along (1010) plane, which indicates the pure hcp structure and well-preferred growth of Co nanowires along [1010] direction at temperatures of $45^{\circ} \mathrm{C}$, and $60^{\circ} \mathrm{C}$.

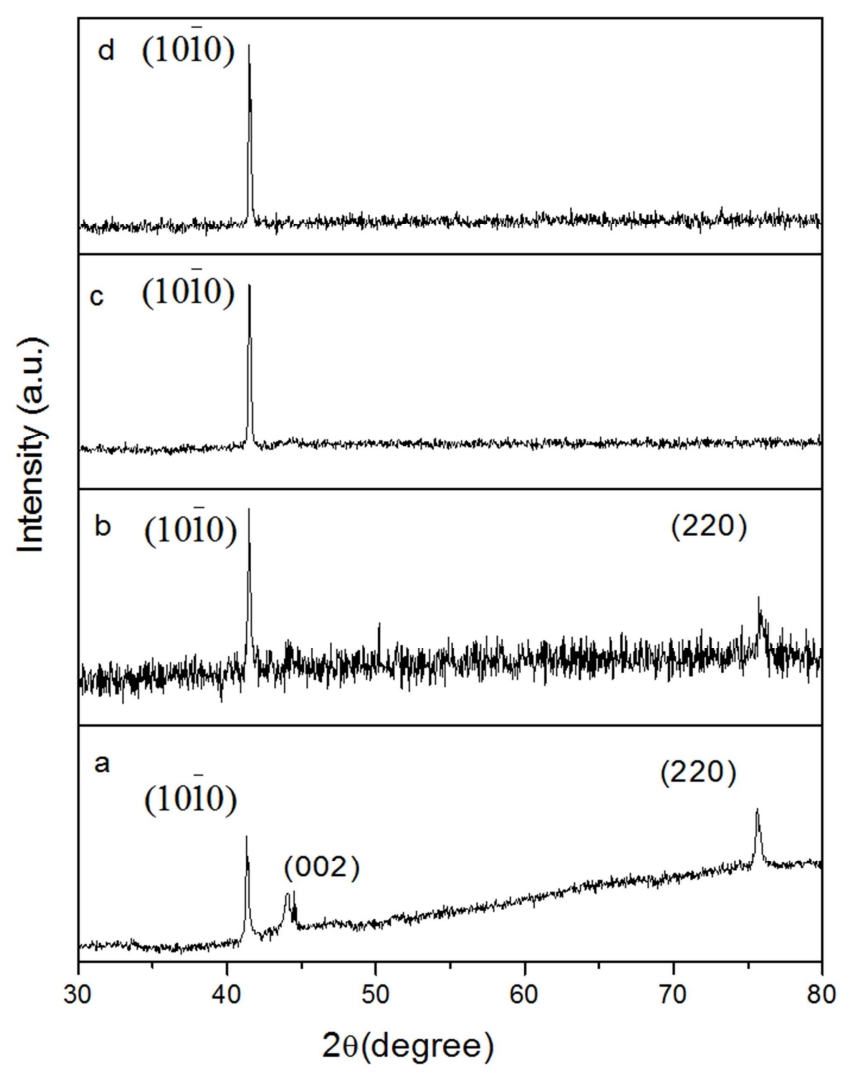

Figure 7. XRD pattern for electrochemically deposited Co nanowires in the pores of $A A O$ template at different temperatures having concentration of $0.35 \mathrm{M}$, a) $25^{\circ} \mathrm{C}$, b) $35^{\circ} \mathrm{C}$, c) $45^{\circ} \mathrm{C}$, and d) $60^{\circ} \mathrm{C}$.

In order to understand the effect of solution concentration and the deposition temperature, the X-ray diffraction results are gathered in Table 2. From the table one can see three points. Firstly, the structure of the deposited Co nanowires is fcc at low temperature $\left(<25^{\circ} \mathrm{C}\right)$ for all concentrations. Secondly, for all concentrations high temperatures $\left(>25^{\circ} \mathrm{C}\right)$ favor the growth in hep phase. Moreover, higher temperature favors hep phase while higher concentration favors fcc phase.

\section{Discussion}

From the dependence of the Co crystal structure on the size of a Co nucleus, we can obtain insight into the mechanism of forming fcc $\mathrm{Co}$ in electrodeposition. In the following, first explain the dependence of the Co crystal structure on the size of Co nucleus and then elucidate the mechanism for formation of fcc Co crystals in electrodeposition at ambient temperature.

\subsection{The Dependence of the Crystal Structure of Co Nucleus on Its Size}

The total free energy of a crystal nucleus is the sum of the bulk free energy and surface free energy of the nucleus. The bulk free energy of hcp Co $\left(G_{h c p}\right)$ is lower than that of fcc Co 
$\left(G_{f c c}\right.$ ) at temperatures below $420^{\circ} \mathrm{C}$. The difference between $G_{h c p}$ and $G_{f c c}$ is of the order of $10^{6} \mathrm{~J} / \mathrm{m}^{3}$. [22, 27] However, the surface energy density of hcp Co phase is higher than that of fcc Co phase. $[22,28,29]$ At temperatures below $420^{\circ} \mathrm{C}$, therefore, the crystal structure of Co nucleus can depend on the size. When the size of a Co nucleus is very small, the fcc structure can be thermodynamically more stable than the hcp structure due to the significant surface effect. This has been confirmed by the many experimental observations that Co particles with very small sizes are fcc phase. [20, 21, 30, 31] For example, when sputtering Co onto a substrate under a low argon gas pressure of 0.15 Torr, Co particles grown on the substrate are fcc structure. TEM results show that these fcc Co particles have sizes of 10-20 $\mathrm{nm}$. [19, 22] However, when sputtering Co in a higher argon gas pressure, hcp-Co particles are obtained. TEM results show that these hep-Co particles have sizes of 40-50 nm. [19, 22] These experimental results can be explained as follow. In the former case, smaller nuclei of fcc Co form. Once the smaller nuclei of fcc Co form, they grow into smaller fcc Co particles $(10-20 \mathrm{~nm})$. In the latter case, larger nuclei of hcp Co form, they grow into larger hcp Co particles (40-50nm). From the above arguments, it is clear that a larger Co nucleus favors an hcp structure while a smaller Co nucleus favors an fcc structure.

\subsection{The Effect of Deposition Potentials and $\mathrm{pH}$ on the Crystal Structure of Co}

In the electrodeposition for formation of metal nanowires, hydrated metal ions in solution arrive at the surface of a metal and are neutralized by electron tunneling, forming neutral adatoms at the surface. [23] These adatoms can move and form a cluster of $\mathrm{N}$ adatoms. According to the classical electrochemical nucleation theory, the free energy of formation of a 3 dimension cluster of $N$ adatoms, $\Delta G(N)$, is given by $[32,33]$.

$$
\Delta G(N)=-N z e \eta+b N^{\frac{2}{3}}
$$

where $z$ is the valence of the hydrated metal ions, $e$ is the elementary electric charge, $\eta$ is the overpotential, $b$ is the constant depending on the geometrical shape of the cluster. Taking the derivative of Eq. (1) with respect to $N$ and equating to zero yields the size of the critical cluster, $N_{\mathrm{c}}$

$$
N_{c}=\left(\frac{2 b}{3 z e \eta}\right)^{3}
$$

From eq. (2), it can be seen that the size of the critical cluster $N_{\mathrm{c}}$ depends on both overpotential $(\eta)$ and the geometrical shape of the cluster $(b)$. The size of the critical cluster decreases with increasing the overpotential. The physical meaning of the critical cluster is that the critical cluster can be either transformed into a crystal nucleus for growth if gaining one atom or dissolved if losing one atom. [33] Before transformation into a crystal nucleus, the structure of the critical cluster can be like amorphous and not well defined.
The formation of clusters of Co atoms occurs on a polycrystalline $\mathrm{Au}$ surface. The surface structure of the polycrystalline $\mathrm{Au}$ is not smooth but geometrically irregular in the nanoscale. This leads to clusters formed on the $\mathrm{Au}$ surface having various shapes. It can therefore be seen from eq.(2) that the sizes of the critical clusters $N_{\mathrm{c}}$ formed under a fixed deposition potential should have a distribution because of different shape factors $(b)$. At the low potential of $-1.6 \mathrm{~V}$, the sizes of all critical clusters, which are determined by eq. (2), are large. This favors formation of the large crystal nuclei of hcp Co for the growth. As a result, pure hcp Co nanowires were observed. When the potential value increases to $-2.0 \mathrm{~V}$, the size distribution of critical clusters shifts to small sizes, in which a small fraction of critical clusters is small in size enough to form crystal nuclei of fcc Co. Thus, a small fraction of fcc Co crystals formed in nanowires. For deposition at $-2.5 \mathrm{~V}$, the fraction of fcc Co nuclei increases relatively since the size distribution of critical clusters shifts further to smaller sizes; so the fraction of $\mathrm{Co}$ fcc crystals formed at $-2.5 \mathrm{~V}$ increases relatively. At deposition at $-3.0 \mathrm{~V}$, the sizes of all the critical clusters is in the range of forming fcc Co nuclei and therefore pure fcc Co nanowires were observed.

The $\mathrm{pH}$ effect can also be explained by the mechanism described above. The environment of nucleation sites for deposition of Co films is significantly different from that of Co nanowires. The size of metal grains in the substrate for deposition of Co films is of the order of micrometers. The sizes of facets of the grains are also of the order of micrometers. The surface of the substrate consists of two parts: (1) the areas located in boundaries of grains, and (2) the areas located in facets of grains. Since the areas of facets are dominated over the surface, nucleation of Co atoms can occur mainly on the facets. A positively charged hydrogen ion within an acidic electrolyte solution is hydrated, forming a hydroxonium ion $\mathrm{H}_{3} \mathrm{O}^{+}$. The hydroxonium ion in the bulk of the solution can diffuse to the cathodic surface where neutralization (dehydration) occurs by electron tunneling from the cathodic metal surface to hydroxonium ion, forming the neutral hydrogen atom. [33] The produced hydrogen atoms can be divided into four parts. First, hydrogen atoms can be incorporated into lattice. This part is an extremely small fraction as the probability of hydrogen dissolved into the lattice is extremely small. [1] Second, hydrogen atoms can be incorporated into grain boundaries as the grain boundaries, in which the metal atoms are loosely packed, have much space to accommodate hydrogen atoms. Moreover, the hydrogen can penetrate through the substrate by diffusing along the grain boundaries and desorb into air; and this can be understood by the experimental observation that the hydrogen in air can penetrate through the stainless steel wall of the vacuum chamber and go into the chamber. However, this part is also a very extremely fraction because of the very extremely volume of grain boundary regions compared to the lattice volume. Third, the hydrogen atoms can recombine to form adsorbed hydrogen molecules, which can desorb from the deposited surface as bubbles. Fourth, hydrogen atoms can occupy the surface sites and impede Co atoms from diffusing to 
nucleation sites when hydrogen atoms are large in number. [34] This can have an effect on the shape and size of clusters. In the case of low $\mathrm{pH}$, hydrogen atoms at the surface can be large in number enough to impede Co atoms from diffusing to nucleation sites, as shown in Fig. 8. These $\mathrm{H}$ adatoms can change the shape of clusters and reduce the size of clusters, which can lead to formation of smaller clusters and so fcc Co nuclei. Therefore low $\mathrm{pH}$ favors formation of fcc Co films.

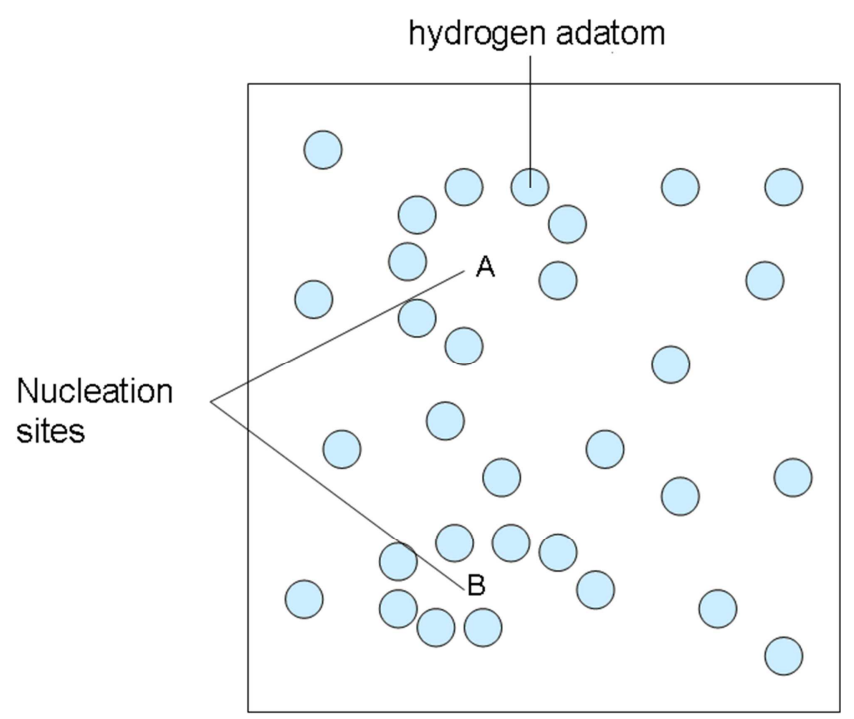

Figure 8. Schematic illustration of the effect of $H$ adatoms on the shape and size of Co clusters. The shape and size of the cluster formed at site $A$ is different from that at site B. Not drawn to scale.

For deposition of Co nanowires, however, nucleation occurs on the sputtered $\mathrm{Au}$ film, which consists of $\mathrm{Au}$ nanoparticles. There are much more grain boundaries in the $\mathrm{Au}$ film than in the substrate for deposition of Co film. For the sputtered $\mathrm{Au}$ film, the volume of grain boundary regions is appreciable due to the significant surface effect, compared to that of lattice. Moreover, the thickness of Au film is small (about 150-200nm). In this case, a lot of hydrogen adatoms can quickly go into the grain boundaries, then penetrate through the thin $\mathrm{Au}$ film by diffusing along the grain boundaries and desorb into air, as argued above. As a result, $\mathrm{H}$ adatoms on the surface of $\mathrm{Au}$ film can be significantly reduced in number so that cannot impede Co atoms from diffusing to nucleation sites. Thus, hydrogen atoms cannot influence the shape and size of clusters and therefore no $\mathrm{pH}$ effect occurs in deposition of Co nanowires.

The fcc Co nanowires of $50 \mathrm{~nm}$ in diameter can be metastable with respect to the hcp Co nanowires at room temperature. This means that the free energy of the fcc Co nanowires can be higher than that of the hcp Co nanowires. However, the transformation from fcc phase to hcp phase requires overcoming an energy barrier and thermal energy at room temperature is too small to activate the transformation. The fcc structure of Co nanowires therefore remain unchanged at room temperature.

\subsection{The Effect of Deposition Temperature and Electrolytic Cell Concentration on the Crystal Structure of Co}

When electrodeposition originates, nucleation must arise. This situation is just related to the nucleation which happens in a supersaturated solution [35]. Here an ion go into the diffusion layer, dehydrated, and after discharging it must diffuses and ultimately form a nucleus by linking other ad-atoms. The nucleation rate depends on the average number of critical clusters (n) and the diffusion of molecules to the cluster $(\beta)$, is given by the equation

$$
I=n \beta
$$

The average population of the critical nuclei is given by the relation

$$
n \propto \exp \left(-\Delta G / k_{B} T\right)
$$

Where $\Delta G$ is the critical free energy needed corresponding to that of critical nucleus, $k_{B}$ is the Boltzmann constant, and $\mathrm{T}$ is the temperature. The number of clusters of a certain size formed is a function of the total number of atoms in the system, the free energy to create a cluster, and the temperature. The number of clusters increases with increasing temperature. The crystal structure of Co nucleus can depend on the size. When the size of a Co nucleus is very small, the fcc structure can be thermodynamically more stable than the hop structure due to the significant surface effect. Many researches confirmed by experimental observations that $\mathrm{Co}$ particles with very small sizes are fcc phase $[20,21,30,31]$. From the relation the nucleation probability will be high if temperature of electrolyte is increased. On the higher nucleation rate the size of the nucleus is small which results in the hcp phase as discussed above. The same phenomenon is seen in our case, higher temperature causes the higher nucleation rate result into hcp phase and lower temperature favor the smaller nuclei to form fcc Co. It is clear from the above arguments that a larger Co nucleus favors an hcp structure while a smaller Co nucleus favors an fcc structure.

Similarly the impact of the electrolyte concentration on the morphology and on the configuration of the deposited Co nanowires is illustrated in Table 2. The conclusions, which observed upon equating the variations in the surface morphology and the structure of the deposited cobalt coatings, are the following: As concentration of electrolyte is increased, the size of the growing cobalt nucleus is decreased which means we get fcc phase considerably. We also find the coexistence of both hcp and fcc phases for Co nanowires at different concentration but at different temperature for each concentration. It means temperature and concentration effect are interlink.

Table 2. XRD results for all concentrations and temperatures presenting the phase change.

\begin{tabular}{llllll}
\hline \multirow{2}{*}{ Concentrations } & \multicolumn{2}{l}{ Temperatures } & & & \\
\cline { 2 - 5 } & $5^{\circ} \mathrm{C}$ & $\mathbf{2 5}^{\circ} \mathrm{C}$ & $\mathbf{3 5}^{\circ} \mathrm{C}$ & $\mathbf{6 5}^{\circ} \mathrm{C}$ & $\mathrm{C}$ \\
\hline
\end{tabular}




\begin{tabular}{lllllll}
\hline \multirow{2}{*}{ Concentrations } & Temperatures & & & & & \\
\cline { 2 - 7 } & $\mathbf{5}^{\circ} \mathbf{C}$ & $\mathbf{1 5}^{\circ} \mathbf{C}$ & $\mathbf{2 5}^{\circ} \mathbf{C}$ & $\mathbf{3 5}^{\circ} \mathbf{C}$ & $\mathbf{4 5}^{\circ} \mathbf{C}$ & $\mathbf{6 0}^{\circ} \mathbf{C}$ \\
\hline $0.71 \mathrm{M}$ & $\mathrm{fcc}$ & $\mathrm{fcc}$ & $\mathrm{fcc}$ & hcp and fcc & hcp and fcc & hcp and fcc \\
$0.53 \mathrm{M}$ & - & $\mathrm{fcc}$ & $\mathrm{fcc}$ & $\mathrm{hcp}$ and fcc & hcp & hcp \\
$0.35 \mathrm{M}$ & - & - & hcp and fcc & hcp and fcc & hcp & hcp \\
\hline
\end{tabular}

\section{Conclusions}

The formation of fcc Co nanowires depends mainly on the deposition potential. Crystals of fcc Co appear in the nanowires at $-2.0 \mathrm{~V}$ and the fraction of fcc Co crystals increases with increasing the deposition potential; and at $-3.0 \mathrm{~V}$ the nanowires are pure fcc Co. It is also concluded that growth orientation of Co nanowires prominently depends on the electrolytic concentration and temperature. When the concentration of electrolytic cell is high, fcc Co structure is dominant in XRD results at nearly room temperature $\left(25^{\circ} \mathrm{C}\right)$ and below, whereas at low concentration this dominance of fcc Co structure is gradually decreased. It has also been seen that under low temperature of electrolytic cell of different concentration Co nanowires exhibits fcc phase, however fcc phase changes to hcp phase when temperature is increased. Simply, high concentration and low temperature of electrolytic cell favor fcc phase whereas low concentration and high temperature favor hcp phase of Co nanowires. The $\mathrm{pH}$ of the solution has little effect on formation of fcc Co nanowires. The formation of fcc Co crystals can be attributed to smaller critical clusters formed at higher deposition potentials since the smaller critical clusters favor forming fcc nuclei. The critical clusters formed at a fixed potential can have a distribution in size and the size distribution can shift to smaller sizes when the potential value increases. At $-1.6 \mathrm{~V}$, the sizes of all critical clusters can be large enough to form hcp nuclei. At $-2.0 \mathrm{~V}$ and $2.5 \mathrm{~V}$, a part of critical clusters can be smaller in size and can be transformed into fcc nuclei. At $-3.0 \mathrm{~V}$, all critical clusters can be small in size enough to form fcc nuclei.

\section{Acknowledgment}

This work is financially supported by a research grant from Wuhan University of Science and technology.

\section{References}

[1] Nakahara S, Mahajan S. The Influence of Solution p H on Microstructure of Electrodeposited Cobalt. J Electrochem Soc. $1980 ; 127283$.

[2] Cohen-Hyams T, Kaplan WD, Yahalom J. Structure of Electrodeposited Cobalt. Electrochemical and Solid-State Letters. 2002; 5: C75-C8.

[3] Vicenzo A, Cavallotti PL. Growth modes of electrodeposited cobalt. Electrochimica Acta 2004; 49: 4079-89.

[4] Kersten H. Physics. 1932; 2: 274.

[5] Cadorna L, Cavallotti P. Electrochim Metall. 1966; 1: 364.
[6] Sard R, Schwartz CD, Weil R. J Electrochem Soc. 1966; 113 : 424.

[7] Sadana YN. Surf Technol 1977; 5: 97.

[8] Wang XW, Fei GT, Tong P, Xu XJ, Zhang LD. Structural control and magnetic properties of electrodeposited Co nanowires. Journal of Crystal Growth. 2007; 300: 421-5.

[9] Huang XH, Li L, Luo X, Zhu XG, Li GG. Orientation-Controlled Synthesis and Ferromagnetism of Single Crystalline Co Nanowire Arrays. J Phys Chem C. 2008; 112: $1468-72$.

[10] Li F, Wang T, Ren L, Sun J. Structure and magnetic properties of Co nanowires in self-assembled arrays. Journal of Physics: Condensed Matter. 2004; 16: 8053.

[11] Ramazani A, Kashi MA, Alikhani M, Erfanifam S. Optimized microstructure and magnetic properties in arrays of ac electrodeposited Co nanowires induced by the continuous and pulse electrodeposition. Journal of Physics D: Applied Physics. 2007; 40: 5533.

[12] Zhang J, Jones GA, Shen TH, Donnelly SE, Li G. Monocrystalline hexagonal-close-packed and polycrystalline face-centered-cubic Co nanowire arrays fabricated by pulse dc electrodeposition. Journal of Applied Physics. 2007; 101: 054310 .

[13] Wang XW, Fei GT, Tong P, Xu XJ, Zhang LD. Structural control and magnetic properties of electrodeposited Co nanowires. Journal of Crystal Growth. 2007; 300: 421.

[14] Darques M, Encinas A, Vila L, Piraux L. Tailoring of the c -axis orientation and magnetic anisotropy in electrodeposited Co nanowires. Journal of Physics: Condensed Matter. 2004; 16 : S2279.

[15] Pan H, Liu B, Yi J, Poh C, Lim S, Ding J, et al. Growth of Single-Crystalline Ni and Co Nanowires via Electrochemical Deposition and Their Magnetic Properties. The Journal of Physical Chemistry B. 2005; 109: 3094-8.

[16] Han X, Liu Q, Wang J, Li S, Ren Y, Liu R, et al. Influence of crystal orientation on magnetic properties of hep Co nanowire arrays. Journal of Physics D: Applied Physics. 2009; 42: 095005 .

[17] Wang XW FG, Xu XJ, Jin Z, Zhang LD. Size-dependent orientation growth of large-area ordered Ni nanowire arrays. J Phys Chem B. 2005; 109: 24326-30.

[18] Granqvist CG, Buhrman RA. Ultrafine metal particles. J Appl Phys. 1976; 47: 2200.

[19] Kitakami O, Sato H, Shimada Y, Sato F, Tanaka M. Size effect on the crystal phase of cobalt fine particles. Physical Review B. 1997; 56: 13849.

[20] Gangopadhyay S, Hadjipanayis GC, Sorensen CM, Klabunde KJ. IEEE Trans Magn 1992; 28: 3174.

[21] Kimoto K, Kamiya Y, Nonoyama M, Ueda R. Jpn J Appl Phys. 1963; 2: 702 . 
[22] Sato H, Kitakami O, Sakurai T, Shimada Y. Structure and magnetism of hcp-Co fine particles. J Appl Phys 1997; 81: 1858.

[23] Tan M, Chen XQ. Growth Mechanism of Single Crystal Nanowires of fcc Metals ( $\mathrm{Ag}, \mathrm{Cu}, \mathrm{Ni}$ ) and hep Metal (Co) Electrodeposited. Journal of The Electrochemical Society. 2012; 159: K15.

[24] Williams DB, Carter CB. Transmission Electron Microscopy, A Textbook for Materials Science. New York Springer 2009.

[25] Cho JU, Wu JH, Min JH, Ko SP, Soh JY, Liu QX, et al. Control of magnetic anisotropy of Co nanowires. Journal of Magnetism and Magnetic Materials 2006; 303: e281.

[26] Huang, Li L, Luo X, Zhu, Li. Orientation-Controlled Synthesis and Ferromagnetism of Single Crystalline Co Nanowire Arrays. The Journal of Physical Chemistry C. 2008; 112: 1468-72.

[27] Nishiyama Z. Martensitic Transformation. New York: Academic; 1978.

[28] Miedema AR, Nieuwenhuys BE. Surf Sci. 1981; 104: 491.
[29] Alden M, Mirbt S, Skriver HL, Rosengaard NM, Johansson B. Phys Rev B 1992; 46: 6303.

[30] Troiano AR, Tokich JL. Trans Metall Soc AIME. 1948; 195: 728.

[31] Owen EA, Jones DM. Proc R Soc London, Ser B. 1954; 67: 456.

[32] Isaev VA, Grishenkova OV. Electrochemistry Communications. 2001; 3: 500 .

[33] Paunovic M, Schlesinger M. Fundamentals of Electrochemical Deposition. New York: Wiley; 1998.

[34] Krause A, Uhlemann M, Gebert A, Schultz L. A study of nucleation, growth, texture and phase formation of electrodeposited cobalt layers and the influence of magnetic fields. Thin Solid Films. 2006; 515: 1694.

[35] WINAND R. Fundamentals and Practice of Aqueous ElectrometallurgyÕÕ (short course) (The Metallurgical Society of CIM, Montreal, PQ, Canada). 1990: 7. 